\title{
Oficina terapêutica como expressão da subjetividade ${ }^{1}$
}

\author{
Izamir Duarte de Farias ${ }^{2}$ \\ Maira Buss Thofehrn ${ }^{3}$ \\ Ana Paula Müller de Andrade ${ }^{4}$ \\ Lisa Antunes Carvalho ${ }^{5}$ \\ Helen Nicoletti Fernandes ${ }^{6}$ \\ Adrize Rutz Porto ${ }^{7}$
}

Objetivo: Conhecer a percepção de profissionais de diferentes formações na área da saúde a respeito do funcionamento das oficinas terapêuticas no CAPS. Pesquisa de abordagem qualitativa, descritiva e exploratória, fundamentada pela teoria Histórico-Cultural de Vygotsky. Foram realizadas entrevistas semiestruturadas, em que participaram vinte e quatro profissionais de saúde dos Centros de Atenção Psicossocial da cidade de Pelotas/RS. As oficinas terapêuticas foram identificadas como espaços que propiciam aos pacientes dos serviços de saúde mental meios de buscar suas potencialidades valorizando os aspectos saudáveis da vida, permitindo a expressão da subjetividade através da arte, do artesanato e das atividades coletivas terapêuticas.

Descritores: Saúde Mental; Serviços de Saúde Mental; Terapia Pela Arte; Enfermagem.

\footnotetext{
${ }^{1}$ Artigo extraído de Dissertação de Mestrado "Oficinas terapêuticas: significado para profissionais de saúde do CAPS", apresentada à Universidade Federal de Pelotas, Pelotas, RS, Brasil

${ }^{2}$ Doutorando, Faculdade de Enfermagem, Universidade Federal de Pelotas, Pelotas, RS, Brasil. Terapeuta, Centro de Atenção Psicossocial Escola, Pelotas, RS, Brasil

${ }^{3} \mathrm{PhD}$, Professor Adjunto, Universidade Federal de Pelotas, Pelotas, RS, Brasil

${ }^{4}$ Pós-doutoranda, Faculdade de Enfermagem, Universidade Federal de Pelotas, Pelotas, RS, Brasil

${ }^{5} \mathrm{MSc}$, Professor, Faculdade Anhanguera, Pelotas, RS, Brasil

${ }^{6}$ Doutoranda, Faculdade de Enfermagem, Universidade Federal de Pelotas, Pelotas, RS, Brasil

${ }^{7} \mathrm{PhD}$, Professor Assistente, Departamento de Enfermagem, Universidade Federal de Pelotas, Pelotas, RS, Brasil

Correspondência:

Helen Nicoletti Fernandes

Universidade Federal de Pelotas, Faculdade de Enfermagem

Rua Gomes Carneiro, 01

Bairro: Porto

CEP: 96010-610, Pelotas, RS, Brasil

E-mail: helyfern@hotmail.com
} 


\section{Therapeutic workshops as expressions of subjectivity}

Objective: Obtain knowledge on the perceptions of professionals with different backgrounds in the health area regarding the therapeutic workshops at CAPS (Centro de Atenção Psicosocial - Psychosocial Care Center) units. The approach of this survey is qualitative, descriptive, and exploratory, grounded in the historic-cultural theory of Vygotsky. Semi-structured interviews were performed, where the participants were twenty-four health professionals from the Psychosocial Care Centers of the city of Pelotas, in the state of Rio Grande do Sul. The therapeutic workshops were identified as spaces that provide patients of mental health services with means of exploring their potentialities, valuing the healthy aspects of life, allowing subjectivity to be expressed through art, craftwork, and collective therapeutic activities.

Descriptors: Mental Health; Mental Health Services; Art Therapy; Nursing.

\section{Oficina terapéutica como expresión de la subjetividad}

Objetivo: Conocer la percepción de profesionales de diferentes formaciones en el área de la salud a respeto del funcionamiento de las oficinas terapéuticas en el CAPS. Investigación de abordaje cualitativa, descriptiva y exploratoria, fundamentada por la teoría HistóricoCultural de Vygotsky. Fueron realizadas entrevistas semiestruturadas, en que participaron veinticuatro profesionales de salud de los Centros de Atención Psicossocial de la ciudad de Pelotas/RS. Las oficinas terapéuticas fueron identificadas como espacios que propician a los pacientes de los servicios de salud mental medios de buscar sus potencialidades valorando los aspectos saludables de la vida, permitiendo la expresión de la subjetividad a través del arte, de la artesanía y de las actividades colectivas terapéuticas.

Descriptores: Salud Mental; Servicios de Salud Mental; Terapia con Arte; Enfermería.

\section{Introdução}

A dimensão da subjetividade não se inclui em um campo puramente racional, mas está relacionada a uma cadeia de significações, nem sempre perceptível para o indivíduo ou para a organização que pertence. Esta dimensão por sua vez está inserida no contexto das relações interpessoais na enfermagem e é entendida como a existência da confiança, da responsabilidade, da ética, da colaboração, da cooperação, do engajamento, da criatividade e da iniciativa. Assim, deve ser trabalhada pela reconstrução coletiva das formas de interação, de comunicação e de ação, para fortalecer tanto o trabalhador, quanto o paciente que procura os serviços de saúde, a partir da reconfiguração da área relacional e das identidades profissionais ${ }^{(1)}$.

Mesmo que o trabalhador esteja imerso em um universo de normas, regras e submissões, suas manifestações deverão ser, permeadas por suas percepções e construções da sua condição de ser humano, singular, portanto, objetivo e também subjetivo. 
O cotidiano dos Centros de Atenção Psicossocial (CAPS), com relação ao cuidado das pessoas em sofrimento ou transtorno mental, com suas necessidades físicas, psicológicas e sociais decorrentes desta patologia, requer dos profissionais um conjunto de habilidades que permitam visualizar estes pacientes na sua individualidade/singularidade, inseridos no seu contexto histórico-social na comunidade e unidade familiar em que vivem. Desse modo, o pensamento crítico reflexivo das equipes em relação ao seu trabalho e o conhecimento destes quanto a subjetividade das relações são importantes, já que influenciam na tarefa profissional, e se caracterizam por um cuidado comprometido, em que aceitar o outro e entendê-lo é fundamental(2).

Ao ser acolhido por um CAPS, o paciente constrói o seu Plano Terapêutico Singular (PTS) junto com a equipe de profissionais local, incluindo uma ou mais oficinas terapêuticas, com a proposta de abrir nova porta para que, na condição de usuário do serviço, esse indivíduo possa resgatar sua percepção de saúde e rever seus conceitos de ser ou estar doente ou saudável, estabelecendo estratégias que possam contribuir para a sua qualidade de vida.

O Plano Terapêutico Singular, constitui-se um instrumento de trabalho que possibilita a esta equipe traçar ações que considerem a dimensão psicossocial, uma vez que o seu foco principal é o indivíduo e o seu contexto social|(3). Isso pode auxiliar no despertar de uma maior ou menor preocupação com sua condição física ou mental, fazendo com que o paciente perceba questões que vão além da medicalização e da doença.

As oficinas terapêuticas podem constituir uma importante ferramenta para canalizar os pensamentos e as projeções desse paciente à produção de algo útil para si e para a coletividade a sua volta, o que poderá levá-lo a um processo de reabilitação psicossocial mais efetivo, no qual ele tenha consciência de que o objetivo do CAPS e das ferramentas disponibilizadas para este processo é a sua reabilitação.

Este estudo teve como proposta olhar para o cuidado em saúde mental, que por muitos anos esteve alicerçado na psiquiatria e no hospital, e que gradativamente está sendo substituído pela soma dos múltiplos conhecimentos que resultam na constituição da equipe interdisciplinar que presta atendimento nos Centros de Atenção Psicossocial (CAPS). Além disso, teve como objetivo compreender o significado das oficinas terapêuticas, enquanto ferramenta mediadora, para profissionais de saúde do CAPS, e conhecer a percepção de profissionais de diferentes formações na área da saúde a respeito do funcionamento dessas oficinas.

A questão que o norteou foi: qual o significado das oficinas terapêuticas, enquanto ferramenta mediadora, para profissionais de saúde do CAPS?

\section{Materiais e Métodos}

O presente estudo teve uma abordagem qualitativa, descritiva e exploratória, fundamentada pela teoria Histórico-Cultural de Vygotsky(4). De acordo com este referencial, o ser humano constrói seus conhecimentos a partir das significações que concebe através das suas interações com o meio no qual está inserido ${ }^{(5)}$. Buscou-se por intermédio dessa pesquisa, apresentar os significados manifestados nas falas dos profissionais de saúde que compõem as equipes interdisciplinares dos CAPS.

A pesquisa foi realizada na cidade de Pelotas, RS. Os dados foram coletados de abril a agosto de 2013 nos seis CAPS II que compõem a rede de saúde mental do município, após a aprovação do projeto dessa pesquisa pelo Comitê de Ética em Pesquisa da Faculdade de Enfermagem da UFPEL, com parecer número 232.387, em 28 de março de 2013.

A coleta dos dados foi realizada por meio de entrevistas semiestruturadas gravadas e posteriormente transcritas. Foram entrevistados vinte e quatro profissionais de saúde de cada CAPS II de Pelotas, de acordo com sua formação acadêmica, sendo: um médico, um assistente social, um psicólogo e um enfermeiro. O objetivo foi representar nesse estudo essas profissões, contando com a participação desses quatro profissionais para cada CAPS. . Eles foram identificados atribuindo-se a letra "P" de profissional seguida do número da entrevista e de siglas que representam cada profissão. Para o médico e psiquiatra utilizou-se MED; para assistente social, AS; para os enfermeiros, ENF e para psicólogos, PSI. Estas siglas também foram seguidas de um número representando a ordem em que a categoria profissional foi entrevistada, ficando cada participante representado conforme o exemplo: P2MED4.

Foram respeitados os princípios éticos para Pesquisas com Seres Humanos, de acordo com o previsto na Resolução no 466/2012 do Conselho Nacional de Saúde, do Ministério da Saúde ${ }^{(6)}$.

Os dados foram analisados segundo a proposta operativa de Minayo ${ }^{(7)}$, a qual recomenda uma análise pautada basicamente em três etapas: ordenação dos dados, classificação dos dados através de leitura horizontal e exaustiva dos textos, leitura transversal, 
posterior construção das categorias temáticas e análise final dos resultados da pesquisa à luz do referencial teórico.

\section{Resultados e discussão}

A partir da análise dos dados, os resultados da pesquisa foram organizados em três unidades temáticas, como segue:

\section{Oficinas terapêuticas como valorização dos aspectos saudáveis}

As intervenções da equipe interdisciplinar são indispensáveis, porém, tratando-se de um objetivo maior e comum a essa equipe, os distintos espaços terapêuticos devem possibilitar uma avaliação ampliada da pessoa, para que possa ser acessada como um todo, percebendo assim suas manifestações diante do coletivo. Alguns profissionais apontaram as oficinas terapêuticas como espaços que propiciam aos participantes dos serviços de saúde mental meios de buscar suas potencialidades, valorizando os aspectos saudáveis da vida:

As oficinas ajudam a gente a ter essa avaliação mais completa da vida da pessoa como um todo, acho que nos atendimentos individuais acaba aparecendo muito a questão da doença (P10MED2).

Acho que o papel da oficina do serviço é importante pra descentralizar o cuidado em saúde, e desmistificar aquela ideia de que cuidado em saúde é feito por profissionais tipo psicólogo, psiquiatra, enfim [...] quando outros profissionais podem proporcionar um cuidado focado na saúde e nas potencialidades do indivíduo, valorizando seus aspectos e manifestações saudáveis e não proporcionar um trabalho focado na doença (P211PSI5).

Obsevando as devidas proporções e características de cada um desses espaços, as oficinas - pela sua proposta de trabalho focado em um objetivo - , promovem ações que estimulam a expressividade, o aprendizado e o potencial de produção de cada paciente.

As oficinas terapêuticas precisam estar inseridas em um processo dinâmico para que evite a inércia institucional e a adoção de posturas solidificadas que pouco reformam. Esses espaços são ferramentas para a produção de vida e precisam continuamente serem problematizados juntamente com os pacientes. Dessa forma, o CAPS contribui para aproximar o paciente das atividades cotidianas de sua vida, constituindo um trabalho pleno de oportunidades e de inclusão social(8).

\section{Oficinas terapêuticas como valorização das capacidades}

Nas falas a seguir, a partir da proposta de cada oficina, o paciente é capaz de buscar na socialização meios de valorização de suas capacidades:

Nos atendimentos, eu acho importante devolver para a pessoa, mostrar que ela não é o tempo inteiro adoecido, é muito mais tempo saudável do que adoecido (P10MED2).

Em geral, o que o usuário demonstra na maior parte do tempo de oficina é seu lado saudável mesmo, pois ele interage, ele se expressa, ele cria [...] (P21PSI5).

A transformação do paciente e o salto qualitativo na sua vida ocorrem quando ele consegue se perceber como um ser humano maior do que sua própria doença e que, além da manifestação de sintomas, pode descobrir ou redescobrir sua potencialidade para superar o sofrimento e, mesmo, aprender com ele. Além disso, o portador de transtorno mental, na condição de pessoa, com todos os seus atributos, pela lógica da reabilitação psicossocial, pode superar seu sofrimento e voltar a ser produtivo para a sociedade, seja pelo seu trabalho ou pela demonstração de suas capacidades $^{(9)}$.

As oficinas terapêuticas com suas propostas de trabalho permitem à pessoa criar, realidade essa afirmada no depoimento de um dos participantes:

Essa capacidade de criação, o ato criativo é que te faz desenvolver tua atenção nos aspectos saudáveis da pessoa. $\mathrm{Na}$ capacidade de criar alguma coisa. Isso é entrar em contato com a saúde. A gente não fica olhando o sujeito doente, a gente fica vendo as pessoas, com o que elas trazem de saúde (P23AS6).

Dessa forma, o ato criativo e a viabilização dessa criatividade são vistos como sinônimos de saúde. Além disso, a importância de construir a valorização dos aspectos saudáveis que envolvem o indivíduo, de modo a desviar o foco do transtorno mental, é citada como uma potencialidade das oficinas terapêuticas:

$\mathrm{Na}$ oficina, a pessoa partilha o espaço com os outros, aprende a dividir e sai daquele pensamento obsessivo que ela tem em relação à doença [...] ela aprende que existem outras coisas para as quais ela pode atentar que lhe podem ser mais úteis do que ficar pensando só na doença e sofrendo com isso. Muitas vezes, o usuário vê que, junto com outros, ele pode fazer coisas maravilhosas e se sente bem com isso, bem melhor (P8ENF3)

O compartilhamento do espaço das oficinas ou espaços de produção criativa é assinalado como uma potencialidade dessa ferramenta para a produção da valorização dos aspectos saudáveis da pessoa, o que, consequentemente, podemos considerar como a produção da saúde propriamente dita. 
Os momentos guiados pelo modelo psicossocial de cuidado e vivenciados nas oficinas, com a certeza da libertação das amarras psicológicas e sociais que assolaram o doente por anos, passam a ser uma possibilidade para a criação, para a vivência e para que o paciente possa ser o que é, liberando seus monstros e seus temores, desconstruindo o conceito de louco como perigoso e abrindo as celas invisíveis para que ele, de fato, seja um ser sociável, sem o estigma da loucura.

As oficinas terapêuticas são espaços de produção de subjetividade, onde há diálogo, interação, reciprocidade e vínculos que permitem a troca entre profissionais e pacientes, permeados pelo respeito às escolhas. É esse movimento em direção à autonomia que nos permite entender que o CAPS é um espaço de produção de pacientes livres para ir e vir. Essa visão é entendida como compatível com o modelo psicossocial de fazer saúde mental(8).

\section{As oficinas terapêuticas como expressão da subjetividade}

Pensar a expressividade humana de maneira não verbal é extremamente subjetivo e amplo, visto do aspecto linguístico, pois muitas e distintas são as possibilidades do ser humano se comunicar e manifestar seus sentimentos e percepções em relação ao mundo no qual está inserido.

Quando se trata de cuidado em saúde, a percepção do profissional e a amplitude de seus conhecimentos acerca dessa habilidade é de grande valia para que, efetivamente $o$ paciente possa ser compreendido quanto as suas necessidades. Absortos pelo cotidiano de trabalho e pelas tarefas que os absorvem como seres sociais, tais profissionais acabam perdendo a capacidade de ler o próximo além das suas palavras, deixando de aferir nas entrelinhas as mais diversificadas manifestações humanas.

A competência aplicada à comunicação coaduna a necessidade de um preparo técnico e humano, a importância do ouvir e a percepção acurada do outro, assim como a possibilidade de utilização desse aprendizado como investimento no autoconhecimento e no respeito ao próximo, revelando que essa a comunicação, quando direcionada ao paciente, deve ocorrer de forma responsável, solidária, humanizada e ética, também terapêutica ${ }^{(10)}$.

A comunicação eficaz é considerada instrumento fundamental para o cuidado integral e humanizado porque, por meio dela, é possível reconhecer e acolher, de maneira empática, as necessidades do paciente. Quando os profissionais de saúde utilizam esse instrumento de forma verbal e não verbal, permitem ao paciente participar das escolhas e dos cuidados específicos relacionados à sua doença, objetivando à obtenção de tratamento digno ${ }^{(11)}$. Nesse processo, há relacionamento e troca de ideias e de conhecimento, geradores de uma nova consciência capaz de produzir mudanças no ser humano e no mundo ${ }^{(12)}$.

Uma vez que vimos à comunicação não verbal apontada como um artifício valioso para a interação e para a atenção humanizada e integral ao paciente dos serviços de saúde mental, a oficina terapêutica pode ser considerada como uma ferramenta mediadora do processo de comunicação entre os sujeitos..

As oficinas terapêuticas exercem um importante papel para que se compreenda mais integralmente o paciente no contexto do CAPS, uma vez que, as propostas de atividades expressivas, proporcionam a oportunidade de manifestação verbal e a comunicação não verbal(13-14).

[...] a oficina de expressão, serve para o usuário poder colocar as coisas que muitas vezes tem dificuldade de se expressar, até de outra forma, de falar, de sentar, de conversar. Tem paciente que vai conseguir fazer isso dessa forma mais corporal mesmo. Então, eu acho que essas oficinas de educação física, dança, funcionam bem. A de musica também, eu acho que poder expressar seus sentimentos, expressar o que já não vem conseguindo fazer a muito tempo (P9MED1).

As atividades terapêuticas nestes espaços podem ser expressivas , físicas, de linguagem [..] de arte livre, que devem possibilitar e ampliar os meios de tratamento e reabilitação dos pacientes, já que estimulam a motricidade, o cognitivo, o afeto, a autoestima, a interação grupal, proporcionando melhor qualidade de vida e utilização dos espaços coletivos dentro da sociedade ${ }^{(15)}$.

Com a arte e o artesanato, as oficinas se constituem num meio de inserção do paciente na sociedade $^{(16)}$, permitindo a sua organização mental, possibilitando a comunicação com outras pessoas e a descoberta de novas alternativas para preencher 0 seu tempo.

A oficina pode proporcionar que o usuário descubra gostos e interesses que muitas vezes não tem oportunidade na vida de experimentar determinadas atividades, que são prazerosas e que estão mesmo mais relacionadas com a questão da arte (P7PSI1).

É pertinente que pensemos nas oficinas como espaços oportunos para o aprendizado e a experimentação de fazeres que, na maioria das vezes, as pessoas em seu cotidiano de cidadãos 
produtivos, não se dedicam ou não se permitem experimentar. Somente quando precisam se afastar dessas atividades cotidianas devido à doença mental e buscam o tratamento em um CAPS, é que encontram nas oficinas, propostas em que ele pode aprender e ensinar, fazendo um intercâmbio de conhecimentos e construindo novos, a partir das relações que estabelecem nesse espaço.

$A$ arte representa um instrumento importante de ressocialização e de inserção individual em grupos, na medida em que propõe um trabalho baseado no agir e no pensar coletivo, respeitando a diversidade, e a capacidade de cada paciente ${ }^{(17)}$. De acordo com a teoria sócio-histórica de Vygotsky, é nesse espaço relacional que o indivíduo constrói suas significações e se constrói como pessoa na sua completude, por meio das mediações que viabilizam a internalização das experiências ${ }^{(4)}$.

Nesse sentido, o fazer artístico trata-se das práticas e do uso de técnicas para o exercício da expressão de ideias e sentimentos que nem sempre se pode manifestar pela palavra, constituindo-se, esse conjunto de meios, como alternativa para a expressividade não verbal(18), o que é considerado na perspectiva vygotskiana como ferramenta mediadora $^{(4)}$. As cores, os sons, os movimentos, ou outros materiais que possam ser usados, permitem a manifestação de dimensões do consciente ou do subconsciente humano que ainda não puderam ser acessados por via da verbalização, ou mesmo, criam oportunidades para a exteriorização de ideias, conforme relatam os pacientes a seguir:

Oficina terapêutica vem, assim, para possibilitar que a pessoa se expresse, que a pessoa ganhe voz (P7PSI1).

O processo terapêutico de oficina faz toda a diferença, porque você está sempre buscando novas atividades para fazer e novas coisas para ajudar o usuário, para possibilitar que ele possa se expressar e possa ser compreendido (P8ENF3).

O espaço terapêutico forma um dispositivo que contribui de modo peculiar para reflexão sobre o que seria essencial para o trabalho nas oficinas terapêuticas - as articulações com o espaço social. Sendo assim, as atividades e trabalhos propostos devem apontar para fora da instituição. As oficinas terapêuticas são dispositivos que devem viabilizar a desinstitucionalização, por isso seu valor enquanto prática que se articula com o que está fora dos muros da instituição. Vale lembrar, que não se trata apenas de trazer o que está lá fora para dentro, mas também de sair da instituição para o espaço público, para a cidade, enfim para o mundo. Neste sentido, uma oficina não deve existir para melhor organizar o funcionamento institucional, simplesmente entretendo os pacientes e tornando-os ocupados com um passatempo $^{(19)}$.

Eu acho que é nas oficinas terapêuticas que as pessoas de fato aparecem como elas são [...] acaba que na oficina terapêutica, a gente consegue, nos casos mais graves, ter uma resposta mais positiva [...] porque a pessoa está ali, livre de qualquer expectativa assim, do outro, elas estão como elas realmente são (P6PSI2).

A oficina possibilita outras formas de expressão para o usuário, que vão além do diálogo na sala de atendimento individual. Eu acho que isso tem uma função importantíssima dentro do CAPS para que se veja como o usuário realmente está (P10MED2).

Por meio dos relatos, percebe-se a manifestação de um conhecimento que vai ao encontro do que foi preconizado nos primórdios da vanguarda reformista da psiquiatria, ou seja, apostar na oficina terapêutica como uma ferramenta que permite a revelação da pessoa, torna-a uma extraordinária tecnologia de cuidado e de atenção ao assistido. A oficina permite compreendermos o paciente efetivamente, na sua completude, incluindo, assim, as dimensões psíquicas mais complexas do ser humano.

A função terapêutica da oficina é dada, de partida, pela própria convivência que ela instaura, através da relação que se estabelece entre oficineiros e pacientes, e principalmente entre os próprios pacientes. Muitos transtornos mentais são marcados pela tendência ao isolamento, pela dificuldade de estabelecer vínculos afetivos e sociais, o que faz com que,mesmo que esse isolamento persista no cotidiano, fora do CAPS, o momento de convivência nas oficinas é de vital importância(19).

Nesse aspecto, o valor construído para oficina refere-se diretamente ao seu potencial para transcender a dimensão da racionalidade, da palavra e do verbalizável, proporcionando ao paceinete a possibilidade de se manifestar por meio de todas as expressões possíveis, levando-o assim a uma maior percepção e consciência de si mesmo.

\section{Considerações finais}

Percebe-se que os profissionais dos CAPS reconhecem a importância das oficinas terapêuticas para a expressão da subjetividade dos pacientes de centros de atenção psicossocial, tendo em vista ser este reconhecido como um espaço promotor de potencialidades e habilidades, permitindo aos pacientes envolvidos a participação ativa no seu processo de reabilitação psicológica e social . 
Sabe-se que a subjetividade transcende o que se vê e o que pode ser palpável, desse modo, fazse necessário uma reflexão de como essas oficinas necessitam de investimento e de compreensão quanto a sua finalidade, não servindo apenas de um ambiente de entretenimento ou diversão como entendido pelo senso comum, mas de um espaço coletivo terapêutico aonde os profissionais dos Centros de Atenção Psicossocial, também promovem um cuidado individual e integral aos seus pacientes.

Espera-se contribuir para uma reflexão da equipe multidisciplinar quanto ao verdadeiro significado das oficinas terapêuticas e sua intervenção na subjetividade dos pacientes atendidos neste espaço, com o propósito de oferecer um cuidado individual em um ambiente terapêutico saudável.

\section{Referências}

1. Thofehrn MB, Amestoy SC, Porto AR, Arrieira ICO, Dal Pai D. A dimensão da subjetividade e no processo de trabalho da enfermagem. J Nurs Health. 2011;1(1):190-8. 2. Bettin AC. Processos inter-relacionais nos CAPS: o caminho para a construção de equipes interdisciplinares com vínculos profissionais saudáveis [dissertação]. Pelotas (RS): Universidade Federal de Pelotas; 2014. $112 \mathrm{p}$.

3. Ministério da Saúde (BR). Centro de Estudo e Pesquisa em Saúde Coletiva. Guia Prático de matriciamento em saúde mental. Brasília; 2011.

4. Rego TC. Vygotsky: uma perspectiva histórico-cultural da educação. 20ª ed. Petrópolis: Vozes; 2009.

5. Vigotsky LS, Lúria AR, Leontiev AN. Linguagem, desenvolvimento e aprendizagem. $6^{a}$ ed. São Paulo: Icone; 1988.

6. Ministério da Saúde (BR). Conselho Nacional de Saúde. Resolução 466, de 12 de dezembro de 2012: diretrizes e normas regulamentadoras de pesquisa envolvendo seres humanos. Brasília; 2012.

7. Minayo MCS. O desafio do conhecimento: pesquisa qualitativa em saúde. 13ª ed. São Paulo: Hucitec; 2011.

8. Pinho LB, Kantorski LP, Wetzel C, Shwartz E, Lange

C, Zilmer JCV. Atividades terapêuticas: compreensão de famílias e profissionais. Esc Anna Nery. 2013 jul/ set;17(3):534-41.

9. Leão A, Barros S. Inclusão e exclusão social: as representações sociais dos profissionais de saúde mental. Interface-Comunic, Saude, Educ. 2011;15(36):137-52.

10. Ferreira JA, Silva JMB, Soares CCD. Comunicação terapêutica no contexto da atenção a saúde do homem. Cuidado é Fundamental. 2014 jan/mar;6(1):333-43.
11. Mullan BA, Kothe EJ. Evaluating nursing communication skills training course: the relation ships between seff-rated ability satisfaction and actual performance. Nurs Educ Pract. 2010;10(6):374-8.

12. Araújo MMT, Silva MJP. Estratégias de comunicação utilizadas por profissionais de saúde na atenção à pacientes sob cuidados paliativos. Rev Esc Enferm USP. 2012:46(3):626-32.

13. Mendonça TCP. As Oficinas na Saúde Mental: Relato de uma Experiência na Internação. Psicologia, Ciência e Profissão. 2005;25(4):626-35.

14. Lima EMFA. A saúde mental nos caminhos da terapia ocupacional. O Mundo da Saúde. 2006 jan/ mar;30(1):117-22.

15. Kantorski LP, Coimbra VCC, Demarco DA, Eslabão AD, Nunes CK, Guedes AC. A importância das atividades de suporte terapêutico para o cuidado em um Centro de Atenção. Rev Enferm Saúde. 2011 jan/mar;1(1):4-13.

16. Mielke FB, Kantorski LP, Jardim VMR, Olschowsky A, Machado MS. O cuidado em saúde mental no CAPS no entendimento dos profissionais. Ciênc Saúde Coletiva. 2009;14(1):159-64.

17. Azevedo DM, Miranda FAN. Oficinas terapêuticas como instrumentos para recuperação psicossocial. Esc Anna Nery. 2011 abr/jun;15(2):339-45.

18. Silva TMD, Vargas PL. O lúdico e a aprendizagem da pessoa com deficiência visual. Revista da Pósgraduação: Desafios Contemporâneos. 2014;1(1).

19. Pinto VAM. Oficinas Terapêuticas na Saúde Mental: um olhar na perspectiva dos usuários do CAPS [dissertação] . Rio de Janeiro: Universidade Federal do Rio de Janeiro; 2011. 